\title{
UNITARY WHITEHEAD GROUP OF CYCLIC GROUPS
}

\author{
BY MAN KEUNG SIU
}

Communicated by Dock S. Rim, June 7, 1972

In topology, one encounters certain groups known as surgery obstruction groups, introduced by C. T. C. Wall [7]. They can be described in a purely algebraic setting, and so hopefully be computed by algebraic means, notably by techniques developed by $\mathrm{H}$. Bass $[\mathbf{1}]$, which has come to be known as algebraic $K$-theory. This note aims at applying such techniques to the computation of the so-called unitary Whitehead groups, certain quotients of which are the Wall's surgery groups mentioned above. We only state the results and sketch the proof of the main theorem. Details will appear elsewhere. This work constitutes part of Chapter I of the author's dissertation [6] submitted to Columbia University. I am deeply indebted to my adviser, Professor Hyman Bass, for his extraordinary patience, generous help and inspiring guidance.

A unitary ring is a triple $(A, \lambda, \Lambda)$, where $A$ is a ring with involution denoted by $a \mapsto \bar{a}, \lambda$ is an element in the center of $A$ satisfying $\lambda \bar{\lambda}=1$, and $\Lambda$ is an additive subgroup of $A$ satisfying the conditions

and

$$
S_{-\lambda}(A)=\{a-\lambda \bar{a} \mid a \in A\} \subset \Lambda \subset\{a \in A \mid a+\lambda \bar{a}=0\}=S^{-\lambda}(A)
$$

\section{$\bar{a} r a \in \Lambda$}

whenever $a \in A, r \in \Lambda$. A morphism $f:(A, \lambda, \Lambda) \rightarrow\left(A^{\prime}, \lambda^{\prime}, \Lambda^{\prime}\right)$ between two unitary rings is a ring homomorphism $f: A \rightarrow A^{\prime}$ satisfying the conditions $f(\lambda)=\lambda^{\prime}, f(\bar{a})=\overline{f(a)}$ for all $a \in A$ and $f(\Lambda) \subset \Lambda^{\prime}$. By an epimorphism (of unitary rings) we mean a morphism with $f(A)=A^{\prime}$ and $f(\Lambda)=\Lambda^{\prime}$. When $A$ has trivial involution (that is, $a=\bar{a}$ for all $a \in A$, so that $A$ has to be commutative), we single out the case $(A, 1,0)$ and call it the orthogonal case. The symbol $U_{2 n}^{\lambda}(A, \Lambda)$ will denote the group of all invertible $2 n \times 2 n$ matrices $\sigma=\left(\begin{array}{ll}\alpha & \beta \\ \gamma & \delta\end{array}\right)$ such that

$$
\sigma^{-1}=\left(\begin{array}{ll}
\delta^{*} & \lambda \beta^{*} \\
\bar{\lambda} \gamma^{*} & \alpha^{*}
\end{array}\right)
$$

where ${ }^{*}$ means conjugate transpose, and $\beta \alpha^{*}, \delta \gamma^{*}$ have diagonal entries in $\Lambda$. The symbol $U^{\lambda}(A, \Lambda)$ will denote the group inj $\lim U_{2 n}^{\lambda}(A, \Lambda)$ with respect to the filtering given by

AMS (MOS) subject classifications (1970). Primary 15A63, 18F25.

Key words and phrases. Surgery obstruction group, unitary ring, involution, unitary Whitehead group, Mayer-Vietoris sequence, cartesian square. 


$$
\left(\begin{array}{ll}
\alpha & \beta \\
\gamma & \delta
\end{array}\right) \mapsto\left(\begin{array}{llll}
\alpha & 0 & \beta & 0 \\
0 & 1 & 0 & 0 \\
\gamma & 0 & \delta & 0 \\
0 & 0 & 0 & 1
\end{array}\right)
$$

The unitary Whitehead group, denoted by $K U_{1}^{\lambda}(A, \Lambda)$, is the commutator quotient group $U^{\lambda}(A, \Lambda) /\left[U^{\lambda}(A, \Lambda), U^{\lambda}(A, \Lambda)\right]$. It can be shown [6] that this group can also be described as the Whitehead group of a certain $(\lambda, \Lambda)$-unitary category. There is a homomorphism $H: K_{1}(A) \rightarrow K U_{1}^{\lambda}(A, \Lambda)$ induced by the association

$$
\alpha \mapsto\left(\begin{array}{cc}
\alpha & 0 \\
0 & \alpha^{*-1}
\end{array}\right) .
$$

The cokernel of $H$ will be denoted by $W_{1}^{\lambda}(A, \Lambda)$. In the orthogonal case, we write $K O_{1}, W O_{1}$ instead of $K U_{1}^{1}, W_{1}^{1}$ respectively.

We are mainly interested in the case $A=Z \pi$, the integral group ring of a group $\pi$, with involution given by $g \mapsto \bar{g}=g^{-1}$ for all $g \in \pi$, and $\lambda=1$ or -1 , and $\Lambda=S_{-\lambda}(Z \pi)$. We shall denote $L_{1}(\pi)=W_{1}^{1}\left(Z \pi, S_{-1}(Z \pi)\right) /\left\langle w_{1}\right\rangle$ where $w_{1}$ is represented by the matrix $\left(\begin{array}{ll}0 & 1 \\ 1 & 0\end{array}\right)$, and denote

$$
L_{3}(\pi)=W_{1}^{-1}\left(Z \pi, S_{1}(Z \pi)\right) /\left\langle w_{-1}\right\rangle
$$

where $w_{-1}$ is represented by the matrix $\left(\begin{array}{cc}0 & 1 \\ -1 & 0\end{array}\right)$. The case $L_{3}(\pi)$ has been extensively investigated by Bass [3], starting from a computation by Lee [5] for the case $\pi$ cyclic of odd prime order. The main result is $L_{3}(\pi)=0$ for $\pi$ finite abelian of odd order. In this note, we attempt to investigate the case $L_{1}(\pi)$.

THEOREM 1. If $\pi$ is cyclic of odd prime power order $p^{r}$, then

$$
W_{1}^{1}\left(Z \pi, S_{-1}(Z \pi)\right)
$$

is a group of order two, generated by $w_{1}$.

COROLlaRy 2. $L_{1}(\pi)=0$ for $\pi$ cyclic of odd prime power order.

COROLlaRY 3. If $\pi$ is cyclic of odd prime power order $p^{r}$, then

$$
K U_{1}^{1}\left(Z \pi, S_{-1}(Z \pi)\right)
$$

has exponent $2 p^{r}$.

Two main tools in the computation are the Mayer-Vietoris sequence associated to a cartesian square and the unitary analogue of Milnor's $K_{2}$ group. For the latter, consider the group $E U^{\lambda}(A, \Lambda)$, which is the subgroup 
of $U^{\lambda}(A, \Lambda)$ generated by matrices of the form $X_{+}(\beta)=\left(\begin{array}{ll}1 & \beta \\ 0 & I\end{array}\right)$ and $X_{-}(\gamma)$ $=\left(\begin{array}{ll}I & 0 \\ \gamma & I\end{array}\right)$. It can be shown [2] that the group $E U^{\lambda}(A, \Lambda)$ is perfect, so that it possesses a universal central covering. The kernel of this universal central covering will be denoted by $K U_{2}^{\lambda}(A, \Lambda)$. In the orthogonal case, $K U_{2}^{1}$ is written $\mathrm{KO}_{2}$ instead.

THEOREM 4. Suppose

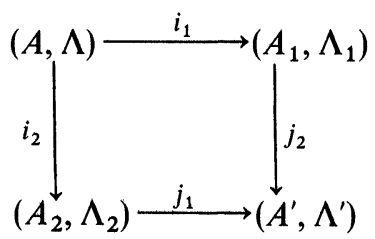

is a cartesian square of unitary rings ( $\lambda$ is same throughout), with $i_{1}, i_{2}, j_{1}, j_{2}$ being epimorphisms of unitary rings. Then there is an exact sequence

$$
\begin{aligned}
& K U_{2}^{\lambda}\left(A_{1}, \Lambda_{1}\right) \oplus K U_{2}^{\lambda}\left(A_{2}, \Lambda_{2}\right) \stackrel{j_{2}-j_{1}}{\longrightarrow} K U_{2}^{\lambda}\left(A^{\prime}, \Lambda^{\prime}\right) \stackrel{\partial}{\longrightarrow} K U_{1}^{\lambda}(A, \Lambda) \\
& \quad \stackrel{\left(i_{1}, i_{2}\right)}{\longrightarrow} K U_{1}^{\lambda}\left(A_{1}, \Lambda_{1}\right) \oplus K U_{1}^{\lambda}\left(A_{2}, \Lambda_{2}\right) \stackrel{j_{2}-j_{1}}{\longrightarrow} K U_{1}^{\lambda}\left(A^{\prime}, \Lambda^{\prime}\right) .
\end{aligned}
$$

This is called the Mayer-Vietoris sequence associated to the cartesian square.

In the computation, we use the following cartesian square $(\lambda=1)$

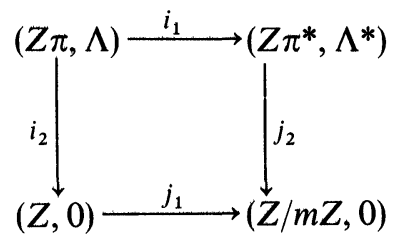

where $Z \pi^{*}=Z \pi /(\Sigma)$ with $\Sigma=\sum_{g \in \pi} g, \Lambda^{*}=S_{-1}\left(Z \pi^{*}\right), \Lambda=S_{-1}(Z \pi)$ and $m=$ order of $\pi$. The homomorphisms $i_{1}, j_{1}$ are the natural epimorphisms, the homomorphism $i_{2}$ is the augmentation map and the homomorphism $j_{2}$ sends $x+(\Sigma)$ to $i_{2}(x)+m Z$. The Mayer-Vietoris sequence associated to the cartesian square is as follows.

$$
\begin{gathered}
K U_{2}^{1}\left(Z \pi^{*}, \Lambda^{*}\right) \oplus K O_{2}(Z) \stackrel{j_{2}-j_{1}}{\longrightarrow} K O_{2}(Z / m Z) \stackrel{\partial}{\longrightarrow} K U_{1}^{1}(Z \pi, \Lambda) \\
\stackrel{\left(i_{1}, i_{2}\right)}{\longrightarrow} K U_{1}^{1}\left(Z \pi^{*}, \Lambda^{*}\right) \oplus K O_{1}(Z) \stackrel{j_{2}-j_{1}}{\longrightarrow} K O_{1}(Z / m Z) .
\end{gathered}
$$

The crux of the work lies in the investigation of the group $K U_{1}^{1}\left(Z \pi^{*}, \Lambda^{*}\right)$. By a certain device, we can pass partially into the $\lambda=-1$ case and obtain the following information. 
Proposition 5. If $\pi$ is cyclic of odd order $m$, then the homomorphism $j_{2}: W_{1}^{1}\left(Z \pi^{*}, \Lambda^{*}\right) \rightarrow W O_{1}(Z / m Z)$ is injective.

Concerning the $K U_{2}^{\lambda}$ groups, we have the following.

PROPOSITION 6. If $\pi$ is cyclic of odd prime power order $m=p^{r}$, then the homomorphism $j_{1}: K_{2}(Z) \rightarrow K O_{2}(Z / m Z)$ is surjective.

Proof of Theorem 1. By Proposition 6, we may replace the group $\mathrm{KO}_{2}(\mathrm{Z} / \mathrm{mZ})$ in the exact sequence $\left({ }^{*}\right)$ by the trivial group. Thus we obtain the following commutative diagram with exact rows:

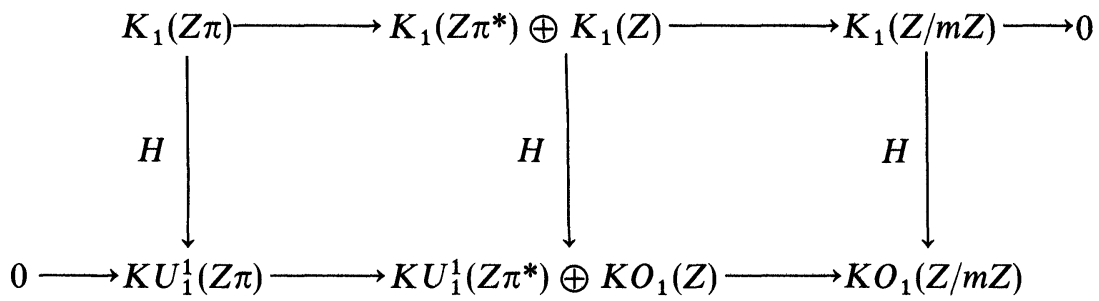

By the Snake Lemma, together with the observation that the homomorphism

$$
\begin{gathered}
\operatorname{ker}\left\{K_{1}\left(Z \pi^{*}\right) \oplus K_{1}(Z) \rightarrow K U_{1}^{1}\left(Z \pi^{*}\right) \oplus K O_{1}(Z)\right\} \\
\rightarrow \operatorname{ker}\left\{K_{1}(Z / m Z) \rightarrow K O_{1}(Z / m Z)\right\}
\end{gathered}
$$

is surjective, we obtain a new exact sequence

$$
0 \rightarrow W_{1}^{1}(Z \pi) \longrightarrow W_{1}^{1}\left(Z \pi^{*}\right) \oplus W O_{1}(Z) \longrightarrow W O_{1}(Z / m Z) .
$$

By Proposition 5, the homomorphism $j_{2}-j_{1}$ restricted to $W_{1}^{1}\left(Z \pi^{*}\right)$ is injective. Thanks to Bass' work [4] on orthogonal groups, we see that $W O_{1}(Z)$ is generated by $w_{1}$. Hence the homomorphism $j_{2}-j_{1}$ restricted to $W_{1}^{1}\left(Z \pi^{*}\right)$ has the same image as $j_{2}-j_{1}$. Hence the kernel of $j_{2}-j_{1}$ is isomorphic to $W O_{1}(Z)$. The result now follows.

\section{REFERENCES}

1. H. Bass, Algebraic K-theory, Benjamin, New York, 1968. MR 40 \#2736.

2. - Unitary algebraic K-theory, Columbia University, New York, 1972 (preprint).

3. - $L_{3}$ of finite abelian groups, Columbia University, New York (to appear).

4. Clifford algebras and spinor norms over a commutative ring, Columbia University, New York, 1972 (preprint).

5. R. Lee, Computation of Wall groups, Topology 10 (1971), 149-176. MR 43 \# 3272.

6. M. K. Siu, Computation of unitary Whitehead group of cyclic groups, Dissertation, Columbia University, New York, 1972.

7. C. T. C. Wall, Surgery of compact manifolds, Academic Press, New York, 1971.

Department of Mathematics, Columbia University, New York, New York 10027 Current address: DePARTMENT OF MATHEMATICS, UNIVERSITY OF MiAmi, CORAL GABles, FLORIDA 33124 